\title{
س
}

$>\mathrm{DE}$

$\approx$ PÉDAGOGIE

\author{
Recherches en éducation
}

180 | juillet-août-septembre 2012

Le CAP : Regards croisés sur un diplôme centenaire

\section{Les déterminants sociaux, scolaires et cognitifs de la réussite en première année universitaire}

The social, educational and cognitive determinants of achievement during the first year at university

Los determinantes sociales, escolares y cognitivos del logro del éxito en primer año universitario

Soziale, schulische und kognitive Faktoren des Erfolgs im ersten und zweiten Hochschulsemester

\section{Sophie Morlaix et Bruno Suchaut}

\section{CpenEdition}

Journals

Édition électronique

URL : http://journals.openedition.org/rfp/3809

DOI : $10.4000 /$ rfp.3809

ISSN : 2105-2913

Éditeur

ENS Éditions

Édition imprimée

Date de publication : 15 juillet 2012

Pagination : 77-94

ISBN : 978-2-84788-388-6

ISSN : 0556-7807

Référence électronique

Sophie Morlaix et Bruno Suchaut, «Les déterminants sociaux, scolaires et cognitifs de la réussite en première année universitaire », Revue française de pédagogie [En ligne], 180 | juillet-août-septembre 2012, mis en ligne le 15 juillet 2015, consulté le 10 décembre 2020. URL : http:// journals.openedition.org/rfp/3809; DOI : https://doi.org/10.4000/rfp.3809 


\section{Les déterminants sociaux, scolaires et cognitifs de la réussite en première année universitaire}

\section{Sophie Morlaix et Bruno Suchaut}

L'objectif principal de cette recherche est de mieux comprendre l'articulation entre les différents facteurs qui agissent sur la réussite des étudiants au terme de la première année à l'université. Une originalité de la démarche est d'intégrer aux modèles explicatifs de la réussite des indicateurs spécifiques des compétences des étudiants à leur entrée à l'université : mesures des performances académiques (niveau en compréhension de l'écrit) et des capacités cognitives. Les analyses mettent en évidence le poids très fort du passé scolaire (retard scolaire, série et mention du baccalauréat) ; le niveau en compréhension de l'écrit et les capacités cognitives ne jouent, à eux seuls, qu'un rôle limité pour expliquer les différences de réussite entre étudiants, l'essentiel de l'influence de ces variables s'étant exprimé auparavant tout au long de la scolarité. En outre, les étudiants pour lesquels le choix de la filière repose sur un projet professionnel réussissent mieux que les autres.

Mots-clés (TESE) : évaluation, enseignement supérieur, compétence, résultats de l'éducation, parcours scolaire.

La question de l'échec dans les premières années de l'enseignement universitaire reste cruciale dans notre pays. L'objectif d'accès de $50 \%$ d'une classe d'âge à un diplôme de l'enseignement supérieur est encore loin d'être atteint et un étudiant sur trois quitte la filière dans laquelle il s'est inscrit à l'issue de la première ou de la deuxième année (Ministère de l'Éducation nationale, 2010). Au fil des années, les publics de l'université sont devenus moins homogènes en termes de capital social, culturel et économique, mais aussi sur le plan de l'âge et de la nationalité. Cette hétérogénéité questionne les chercheurs et plusieurs facteurs explicatifs de l'échec universitaire en première année sont mis en lumière. Pour Alain Coulon (1997), «l'entrée et la réussite dans l'enseignement supérieur relèvent d'un apprentissage, d'une acculturation et ceux qui ne parviennent pas à s'affilier échouent ». Ce processus d'affiliation conditionnerait le passage du statut d'élève à celui d'étudiant et l'acquisition d'une nouvelle identité. Les étudiants qui abandonnent sont, pour l'auteur, ceux pour lesquels la compréhension et l'incorporation des codes de la culture universitaire ne se font pas, ou pas assez vite. Confrontés à leur nouveau métier d'étudiant, certains n'en perçoivent pas les exigences (Beaupère \& Boudesseul, 2009). Les indicateurs d'adaptation à l'université seraient influencés par les caractéristiques initiales de l'étudiant, ces dernières se rapportant aux caractéristiques personnelles d'une part, au passé scolaire d'autre part.

Les travaux qui ont analysé les déterminants de la réussite à l'université mettent tous en évidence 
l'importance de la scolarité antérieure et de l'origine sociale (Beaupère \& Boudesseul, 2009). Les bacheliers de la série scientifique réussissent généralement mieux que les autres; en revanche, les étudiants ayant déjà redoublé dans le secondaire et titulaires d'un baccalauréat technologique ou professionnel présentent plus de risques d'échec (Prouteau, 2009). Les filles réalisent une meilleure insertion universitaire que les garçons, en particulier en raison de leur plus grande adhésion aux règles et aux normes du travail scolaire et de leur meilleure organisation en termes de travail personnel (Frickey \& Primon, 2002). Au-delà de ces facteurs sociaux et scolaires, les conditions dans lesquelles se déroulent les études (notamment le temps de travail individuel de l'étudiant) peuvent aussi être considérées comme des variables liées à la réussite. Par exemple, les travaux du CEREQ et de l'Observatoire national de la vie étudiante (OVE) montrent que le fait de travailler pendant les études peut être bénéfique en termes d'insertion professionnelle, mais constitue a contrario un facteur de risque dans certaines conditions : si la charge horaire est supérieure à un mi-temps, si cette activité s'exerce plus de six mois par an et si l'emploi occupé est éloigné du domaine d'études. En fait, s'il y a concurrence entre l'activité rémunérée et les études, le risque d'échec est plus grand (Béduwé \& Giret, 2004).

La prise en compte d'autres facteurs, de nature plus contextuelle, peut également expliquer cet échec en fin de première année comme le souligne Marie Duru-Bellat (1995). Aussi l'impact du contexte universitaire dans lequel l'étudiant évolue doit être considéré avec attention : le site universitaire, les pratiques pédagogiques, les curricula, les dispositifs d'accompagnement sont autant d'éléments à prendre en compte pour expliquer les différences de réussite en fin de première année (Romainville, 2000 ; Michaut, 2003). De façon à favoriser la réussite du plus grand nombre d'étudiants en fin de première année, des politiques variées ont été mises en place dans différents pays (présélection sur la base des aptitudes, modulation des droits d'inscription en fonction des performances des étudiants, instauration de parcours à la carte laissant une plus grande liberté de choix aux étudiants, etc.). La France, dans un premier temps, a centré son action sur les possibilités de réorientations en cours d'année et sur l'aide à apporter aux étudiants, sous la forme de tutorat notamment. Mais les effets semblent mitigés (Danner, 2000 ; Borras, 2011), de par le ciblage difficile des étudiants fragiles. Depuis 2007, de nouveaux dispositifs voient le jour dans le cadre de l'instauration du Plan Réussite en licence (PRL). Mais les recherches montrent que les dispositifs d'accompagnement méthodologique ou pédagogique touchent rarement ceux qui en auraient le plus besoin : ceux qui assistent aux séances sont en effet ceux dont les chances de réussite sont déjà élevées et qui sont déjà en partie familiarisés avec les codes du travail universitaire. Cet écueil est confirmé récemment par les travaux de Rébecca Shankland (2009), qui montrent que les participants ont généralement réussi leurs études secondaires et sont majoritairement issus de familles dont les parents ont fait des études supérieures.

Notre recherche se situe dans le prolongement des travaux cités mais en intégrant une dimension complémentaire comme facteur explicatif de la réussite ${ }^{1}$. L'hypothèse générale associée à notre questionnement est que les étudiants ne disposent pas tous des mêmes chances de réussite et que celles-ci sont affectées par l'influence de variables très personnelles comme leurs capacités cognitives et leur niveau académique. Ces performances, liées, par ailleurs, à d'autres caractéristiques des étudiants (sociales et scolaires) seraient alors partiellement explicatives des difficultés rencontrées par certains au tout début de leur parcours universitaire. Le rôle des capacités cognitives dans la réussite scolaire, mis au jour dans le contexte français au niveau de l'école primaire (Barrouillet, Camos, Morlaix et al., 2008), n'a pas encore fait l'objet de recherches en ce qui concerne l'enseignement supérieur dans notre pays, ce qui n'est pas le cas dans le contexte anglosaxon (Shulruf, Hattie \& Tumen, 2008). Au-delà des capacités cognitives (mémoire de travail, vitesse de traitement de l'information, raisonnement logique), une facette des performances académiques (test de compréhension de l'écrit) des étudiants a été évaluée. L'identification du poids de chaque groupe de facteurs dans la réussite peut livrer des informations utiles sur les chances de succès (ou les risques d'échec) des étudiants en fonction de leurs caractéristiques personnelles.

L'échantillon sur lequel s'appuient nos analyses est constitué d'étudiants inscrits en première année à l'université de Bourgogne (année universitaire 2010-2011). Si, dans l'absolu, il aurait été souhaitable de prendre en compte l'ensemble des filières de l'université, des contraintes matérielles évidentes dans ce type de travail empirique (pour la passation des épreuves notamment) ont conduit à effectuer des choix dans ce domaine. Nous avons souhaité représenter des filières accueillant une population d'étudiants relativement hétérogène en fonction du passé scolaire (série du bac en particulier) et avons 
Figure 1. Structure relationnelle de la réussite en première année universitaire

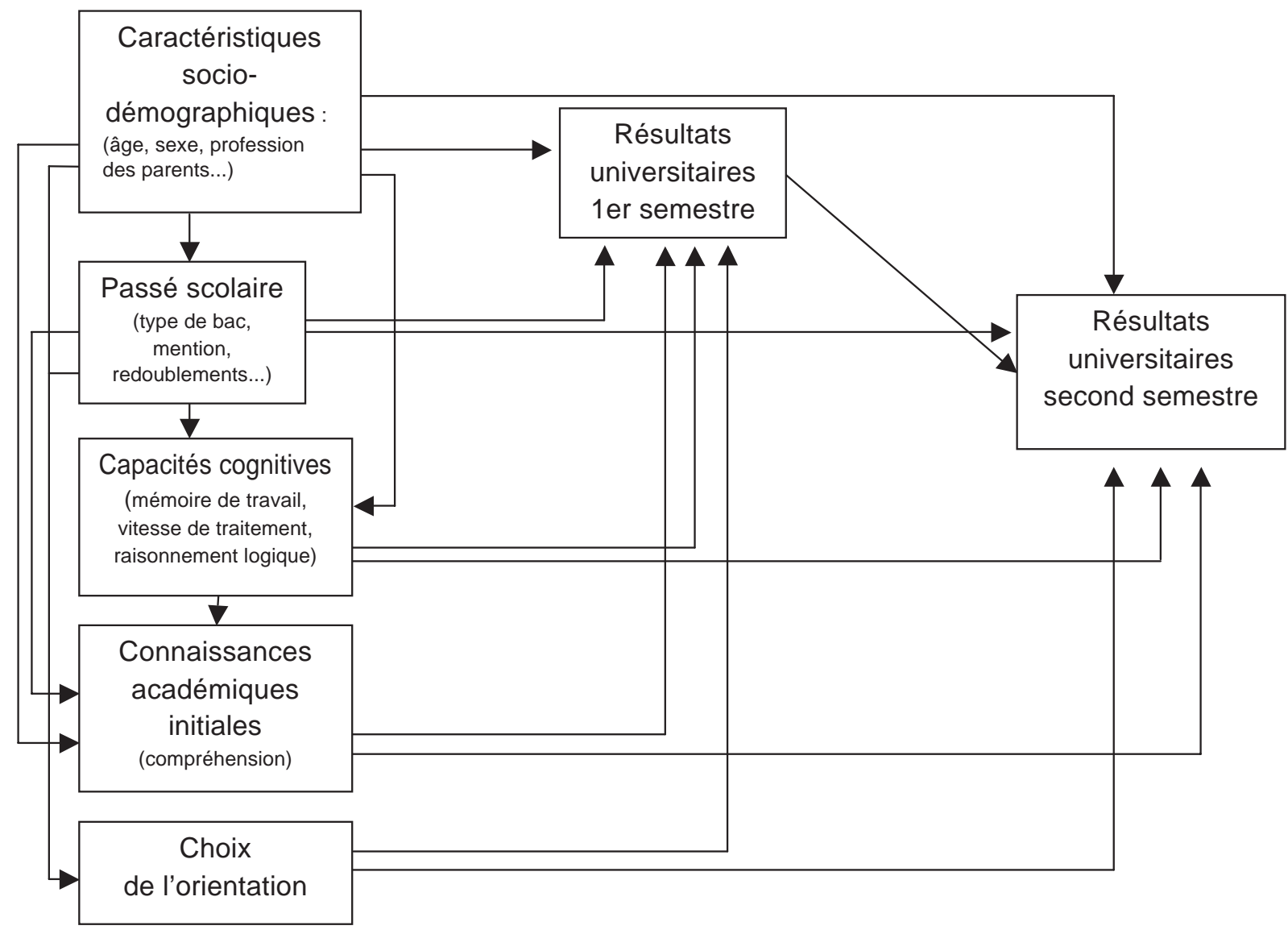

choisi de nous intéresser aux étudiants de première année inscrits en Administration économique et sociale (AES), psychologie et droit. Ces trois filières recensaient 1493 inscrits en septembre 2010, soit $35 \%$ de l'ensemble des étudiants inscrits en première année de l'université de Bourgogne, toutes filières confondues. Le tableau en annexe permet de situer la population de référence de notre échantillon par rapport à l'ensemble des inscrits de première année en fonction de plusieurs caractéristiques des étudiants. Toujours pour des raisons de faisabilité de la recherche, tous les étudiants des trois filières n'ont pu être testés dans les différentes dimensions, 616 étudiants ont été volontaires pour passer les tests de raisonnement logique et de compréhension de l'écrit, ces deux épreuves étant administrées sous une forme "papier-crayon ". Les mesures fines des capacités cognitives ont, quant à elles, porté sur un échantillon plus modeste puisqu'elles nécessitaient des passations en petit groupe (un étudiant par ordinateur) et d'une durée plus longue ; au total, on dispose pour 421 étudiants de l'ensemble des indicateurs de performances académiques et de capacités cognitives. La figure 1 présente l'ensemble des relations statistiques explorées dans cette recherche ${ }^{2}$.

Une mesure des connaissances académiques initiales et notamment celle de la compréhension écrite a été élaborée en utilisant le Diplôme approfondi en langue française (DALF). Des mesures sur trois facettes des capacités cognitives des étudiants ont été effectuées : la mémoire de travail, la vitesse de traitement et enfin le raisonnement. La mémoire de travail est mesurée par trois indicateurs : le Reading 
Tableau 1. Modèle expliquant la variance du score au DALF $(\mathbf{N}=612)$

\begin{tabular}{|c|c|c|c|c|}
\hline \multirow[b]{2}{*}{ Modalité de référence } & \multirow[b]{2}{*}{ Modalité active } & \multicolumn{3}{|c|}{ Modèle 1} \\
\hline & & Coefficient & t de tudent & Prob. \\
\hline \multicolumn{2}{|l|}{ Constante } & $+13,0$ & $+63,6$ & 0,00 \\
\hline \multicolumn{5}{|c|}{ Âge lors de l'obtention du bac } \\
\hline 19 ans ou moins & Plus de 19 ans & $-0,9$ & $-1,92$ & 0,05 \\
\hline \multicolumn{5}{|l|}{ Série du bac } \\
\hline \multirow{2}{*}{ Bac général } & Bac technologique & $-1,0$ & $-2,7$ & 0,00 \\
\hline & Bac professionnel & $-1,6$ & $-2,6$ & 0,00 \\
\hline \multicolumn{5}{|l|}{ Mention au bac } \\
\hline $\begin{array}{l}\text { Pas de mention ou } \\
\text { passable }\end{array}$ & Mention AB, B ou TB & $+1,2$ & $+3,8$ & 0,00 \\
\hline \multicolumn{5}{|l|}{ Profession du père } \\
\hline Autres professions & Cadre & $+0,6$ & $+1,7$ & 0,08 \\
\hline \multicolumn{2}{|l|}{$\mathrm{R}^{2}$} & \multicolumn{3}{|c|}{0,09} \\
\hline
\end{tabular}

Lecture : le recours au modèle de régression permet d'estimer les effets spécifiques de chaque variable en raisonnant « toutes choses égales par ailleurs ". Les estimations statistiques portent sur un effectif de 612 étudiants $(N=612)$. Ainsi, dans le tableau, à âge, série du bac et profession du père donnés, un étudiant qui a obtenu une mention (AB, B ou TB) obtient 1,2 point de plus qu'un étudiant (par ailleurs comparable) qui n'a pas eu de mention. C'est le coefficient de régression qui exprime cet effet; il est associé à un t de Student qui est un indicateur du degré de significativité des coefficients associés à chaque modalité des variables. Plus il est élevé, plus le coefficient a de chances d'être significativement différent de 0 . À ce t de Student correspond une probabilité d'erreur (indiquée « Prob. » dans le tableau). Par exemple, le coefficient de la variable « Mention au bac » est significatif avec un risque d'erreur inférieur à 1 pour $100:$ prob. $=0,00$.

Span, l'Operation Span et l'empan de mémoire de travail issu des épreuves du Time-Based ResourceSharing model (modèle TBRS). La vitesse de traitement est aussi mesurée par trois indicateurs : le Simple Reaction Task (SRT, tâche de détection simple) et deux épreuves adaptées des travaux de Posner (toutes deux des tâches de détection complexes). Ces tests cognitifs ont été adaptés de tests américains comme le Reading Span Task (Daneman \& Carpenter, 1980) ou l'Operation Span Task (Turner \& Engle, 1989), ainsi que les épreuves de vitesse de traitement qui répliquent les tâches de Posner. Les épreuves de mémoire de travail ont été construites selon la structure des tâches informatisées issues du modèle TBRS. Enfin, les capacités de raisonnement sont mesurées par les matrices de Raven. Des mesures individuelles ont été effectuées dans les trois dimensions cognitives pour chacun des étudiants de l'échantillon. D'autres informations ont ensuite été collectées en février suite aux premiers examens universitaires, de façon à recueillir les résultats détaillés des étudiants dans chacune des Unités d'enseignement (UE). De façon conjointe, un questionnaire leur a été proposé se rapportant à leurs conditions d'études (activité rémunérée, méthodes et quantité de travail, motivation, etc.). En fin d'année universitaire, les résultats finaux ont également été collectés.

\section{LES DÉTERMINANTS DES PERFORMANCES ACADÉMIQUES}

Pour la mesure des performances académiques, l'idéal aurait été de disposer d'un test spécifiquement destiné à des étudiants ; en l'absence d'un tel outil, nous avons opté pour une épreuve de compréhension de l'écrit avec le niveau dit «C1 » du DALF. L'épreuve est basée sur un texte de Maupassant d'environ deux mille mots, à partir duquel les étudiants doivent répondre à une dizaine de questions, en commentant ou analysant certains passages. Le DALF est composé de 13 items dans sa partie " compréhension de l'écrit ". Ces items participent à la construction d'un score dont la valeur la plus élevée est de 22 points. Sur l'ensemble de l'échantillon, le score moyen est 
Tableau 2. Modèle expliquant la variance du score au Raven $(\mathbf{N}=\mathbf{6 1 2})$

\begin{tabular}{|c|c|c|c|c|}
\hline \multirow[b]{2}{*}{ Modalité de référence } & \multirow[b]{2}{*}{ Modalité active } & \multicolumn{3}{|c|}{ Modèle 2} \\
\hline & & Coefficient & t de Student & Prob. \\
\hline \multicolumn{2}{|l|}{ Constante } & $+8,1$ & $+31,2$ & 0,00 \\
\hline \multicolumn{5}{|c|}{ Âge lors de l'obtention du bac } \\
\hline 19 ans ou moins & Plus de 19 ans & $-1,3$ & $-3,3$ & 0,00 \\
\hline \multicolumn{5}{|l|}{ Série du bac } \\
\hline Autres bacs & Bac S & $+1,7$ & $+4,9$ & 0,00 \\
\hline \multicolumn{5}{|l|}{ Mention au bac } \\
\hline $\begin{array}{l}\text { Pas de mention ou } \\
\text { passable }\end{array}$ & Mention AB, B ou TB & $+0,5$ & $+1,1$ & 0,09 \\
\hline \multicolumn{5}{|l|}{ Profession du père } \\
\hline Autres professions & $\begin{array}{l}\text { Ouvrier } \\
\text { Agriculteur, artisan, } \\
\text { commerçant } \\
\text { Cadre } \\
\end{array}$ & $\begin{array}{l}+0,8 \\
+0,9 \\
+1,0\end{array}$ & $\begin{array}{l}+2,4 \\
+2,3 \\
+2,8\end{array}$ & $\begin{array}{l}0,02 \\
0,02 \\
0,00\end{array}$ \\
\hline \multicolumn{5}{|l|}{ Mode d'hébergement } \\
\hline Autres modes & En résidence universitaire & $-0,6$ & $-1,7$ & 0,08 \\
\hline \multicolumn{5}{|l|}{ Filière fréquentée } \\
\hline AES et droit & Psychologie & $+1,4$ & $+5,0$ & 0,00 \\
\hline \multicolumn{2}{|l|}{$\mathrm{R}^{2}$} & \multicolumn{3}{|c|}{0,13} \\
\hline
\end{tabular}

de 12,9 avec un écart-type de 3,6 ; la distribution des scores présente une allure gaussienne. Aucune différence de réussite n'est observée entre les filles et les garçons ; il n'y a pas non plus d'écarts en fonction du mode d'hébergement de l'étudiant et entre les étudiants boursiers et les autres ; en revanche, les enfants de cadre affichent des résultats supérieurs aux autres professions (2 points de plus que les enfants d'ouvrier). Les variables liées au passé scolaire sont discriminantes puisque des écarts significatifs sont relevés selon la série du bac (résultats plus faibles pour les bacs technologiques et professionnels), la mention du bac (avantage pour les étudiants ayant obtenu au moins une mention assez bien) et l'âge lors de l'obtention du même diplôme (les élèves affichant deux années de retard ayant des scores significativement inférieurs aux autres). Afin d'obtenir une image globale des déterminants de la réussite au DALF, un modèle de régression a été estimé en ne faisant apparaître que les seules variables significatives (tableau 1). Ce sont le type et la mention du bac qui présentent les coefficients les plus élevés, les élèves ayant obtenu un bac professionnel sont les plus défavorisés dans cette épreuve de compréhension écrite (1,6 point de moins que les étudiants ayant obtenu un bac général). On relèvera enfin que les variables prises en compte expliquent moins de $10 \%$ de la variance du score au $\operatorname{DALF}\left(R^{2}=0,09\right)$.

\section{LES DÉTERMINANTS DES CAPACITÉS COGNITIVES}

Les capacités de raisonnement sont mesurées par les progressive matrices de Raven, la version abrégée administrée aux étudiants comporte 23 «problèmes " à résoudre. Pour chaque situation, 8 vignettes représentant des figures sont présentées au sujet qui doit en trouver les règles de succession et choisir 
Tableau 3. Modèle expliquant la variance des performances en Reading Span $(\mathbf{N}=\mathbf{4 1 8})$

\begin{tabular}{|l|l|c|c|c|}
\cline { 3 - 5 } \multicolumn{2}{l|}{} & \multicolumn{3}{c|}{ Modèle 3 } \\
\hline Modalité de référence & Modalité active & Coefficient & t de Student & Prob. \\
\hline Constante & $+84,7$ & $+40,5$ & 0,00 \\
\hline Garçon & Fille & $-6,2$ & $-3,3$ & 0,00 \\
\hline Non boursier & Boursier & $-4,9$ & $-3,2$ & 0,00 \\
\hline Âge lors de l'obtention du bac & \multicolumn{3}{l}{0,00} \\
\hline 19 ans ou moins & Plus de 19 ans & $-6,3$ & $-3,8$ & 0,0 \\
\hline Filière fréquentée & $-6,8$ & $-2,4$ & 0,00 \\
\hline Psychologie et droit & AES & & 0,08 & \\
\hline$R^{2}$ &
\end{tabular}

parmi 8 autres figures celle qui correspond à la suite de la série. Cette situation de test fait intervenir des aptitudes visuo-spatiales mais aussi la mémoire de travail et la vitesse de traitement de l'information, l'intelligence fluide et l'induction (Huteau \& Lautrey, 1999 ; Jensen, 1987) ainsi que des facteurs spatiaux. Les scores obtenus au Raven sur notre échantillon se distribuent de manière gaussienne et varient de 1 à 20 avec une moyenne de 9,4 et un écart-type de 3,4.

Le tableau 2 expose les estimations d'un modèle expliquant la variance du score au Raven en fonction des caractéristiques des étudiants. Ce modèle explique $13 \%$ des différences de résultats au test et certaines variables comme l'âge à l'obtention du bac, la série et la mention du bac ou encore la profession des parents exercent une influence significative sur les scores. La filière fréquentée, même à caractéristiques des étudiants données, fait des différences puisque les psychologues obtiennent près d'un demi-point de plus que les étudiants d'AES et de droit. Plus surprenant est l'impact du mode d'hébergement au désavantage des étudiants logés en résidence universitaire (la probabilité d'erreur associée au coefficient est toutefois élevée : $8 \%$ ).

Trois mesures de mémoire de travail ont été reconnues comme de meilleurs prédicteurs que celles existant précédemment (Barrouillet, Camos, Morlaix et al., 2008 ; Lépine, Barrouillet \& Camos, 2005). Nous utiliserons ici des épreuves impliquant soit du matériel verbal (Barrouillet, Bernardin, Portrat et al., 2007) soit du matériel visuo-spatial (Barrouillet, Portrat, Vergauwe et al., 2011). La mémoire de travail est mesurée par trois indicateurs : le Reading Span, l'Operation Span et le Time-Based Resource-Sharing model (modèle TBRS). Dans l'épreuve du Reading Span, les sujets doivent mémoriser des chiffres. Entre chaque chiffre, ils lisent des phrases et doivent juger de la plausibilité de ces phrases. Le score obtenu est le pourcentage de chiffres correctement rappelés. Sur l'échantillon, la moyenne du score est de 81 avec un écart-type de 16, il est expliqué à la hauteur de $8 \%\left(R^{2}=0,08\right)$ par les variables prises en compte (tableau 3 ).

Les filles obtiennent des performances inférieures de 6 points à celles des garçons. Ce résultat paraît surprenant dans la mesure où la littérature conclut plutôt à l'absence de différences entre sexes sur cette dimension cognitive. On peut penser que notre résultat est sans doute lié à un biais de sélection; les orientations post-bac étant différenciées sur le plan du genre, le fait de considérer seulement quelques filières de l'enseignement supérieur ne permet pas de généraliser ces différences sexuées à l'ensemble de la population universitaire. Le retard scolaire et le fait d'être boursier sont aussi des caractéristiques qui influent négativement sur les scores des étudiants.

Pour les épreuves relatives à l'Operation Span (modèle non présenté), les sujets doivent mémoriser des lettres. Entre chaque lettre des équations sont présentées et les sujets doivent se prononcer sur la justesse du résultat (vrai ou faux). Les modèles de régression indiquent que certaines tendances relevées dans le modèle précédent (Reading Span) se confirment : moindres performances des filles et des étudiants âgés. D'autres caractéristiques, comme la série du bac (avantage aux scientifiques) ou 


\begin{tabular}{|l|c|c|}
\cline { 2 - 3 } \multicolumn{1}{c|}{} & Raven & Mémoire de travail \\
\hline Mémoire de travail & $+0,23^{* * \star}$ & $-0,24^{* * *}$ \\
\hline Vitesse de traitement & $-0,14^{* * *}$ & \\
\hline
\end{tabular}

Note : ${ }^{* \star *}$ : significatif au seuil de $1 \%$.

le mode de résidence (avantage aux étudiants non hébergés en résidence universitaire) exercent également une influence sur les performances dans ce domaine. Enfin dans le modèle TBRS (non présenté), les sujets doivent mémoriser des lettres et juger de la parité de chiffres. Le pourcentage de variance expliquée par les caractéristiques des étudiants est de l'ordre de $10 \%$; par rapport aux épreuves précédentes, la profession du père contribue elle aussi à l'explication de la variance : avantage aux professions intermédiaires. II faut rappeler qu'à ce niveau d'étude, une sélection sociale a déjà été établie et qu'il est difficile de comparer ces résultats avec ceux habituellement relevés pour les niveaux scolaires inférieurs.

La vitesse de traitement est mesurée par trois indicateurs : le SRT (tâche de détection simple), le Posner 1 et le Posner 2 (tous deux des tâches de détection complexes). Dans la tâche SRT, les sujets doivent appuyer le plus rapidement possible sur la barre espace du clavier à l'apparition d'un carré à l'écran. Les carrés apparaissent 1000 , 2000 ou 3000 ms après le signal. Dans le Posner 1, deux lettres apparaissent côte à côte à l'écran et les sujets doivent repérer si elles sont physiquement identiques. Pour le Posner 2, il s'agit d'une tâche de détection complexe, deux lettres apparaissent côte à côte à l'écran et les sujets doivent repérer si elles sont identiques, qu'il s'agisse de majuscules ou de minuscules. Les données recueillies dans les trois épreuves correspondent à des temps moyens en millisecondes.

Les trois modèles montrent des similitudes dans les pourcentages de variance expliquée qui restent faibles (de 3 à $4 \%$ ), peu de variables semblent donc être discriminantes en ce qui concerne les vitesses de traitement. En ce qui concerne les tâches de traitement simples, on retrouve une tendance négative pour les filles ainsi que pour les étudiants âgés, en revanche les possesseurs d'un bac scientifique apparaissent légèrement plus performants que les autres. Pour le Posner 1, les enfants d'ouvrier réalisent des scores un peu plus élevés que les autres mais c'est encore une fois le genre (à l'avantage des garçons) et la série du bac (au détriment des possesseurs d'un bac professionnel) qui sont les caractéristiques les plus discriminantes. Pour les épreuves du
Posner 2, on notera que les étudiants de psychologie présentent des temps de réaction inférieurs aux autres, c'est aussi le cas des enfants d'ouvrier. Les étudiants boursiers et ceux titulaires d'un bac professionnel affichent des performances plus faibles que celles des non boursiers et des étudiants titulaires d'un bac général ou technologique.

Afin de disposer de mesures plus générales, il peut être pertinent de construire des indicateurs globaux rendant compte des deux dimensions ciblées (mémoire de travail et vitesse de traitement) ; pour nous assurer que ce regroupement soit valide, nous avons calculé des corrélations entre les différentes mesures. Les corrélations sont positives et significatives (au seuil de $1 \%$ ), leur intensité varie toutefois selon les dimensions considérées, la plus forte relation est celle entre les épreuves Posner 1 et Posner $2(+0,63)$. Nous avons donc construit un score moyen en mémoire de travail ainsi qu'un score moyen de vitesse de traitement; chacun de ces scores prenant en compte chacune des performances ${ }^{3}$. Nous disposons ainsi de trois mesures des capacités cognitives : Raven, mémoire de travail et vitesse de traitement. Le tableau 4 indique les corrélations obtenues entre ces trois mesures.

On constate globalement de faibles corrélations ${ }^{4}$, la plus faible étant celle associant le Raven et la vitesse de traitement $(-0,14)$, cela suggère que les trois facettes des capacités cognitives appréhendées dans cette recherche ont une composante autonome à prendre en compte dans l'analyse des déterminants de la réussite.

\section{L'ANALYSE DES FACTEURS EXPLIQUANT LA VARIÉTÉ DES NOTES DU PREMIER SEMESTRE}

Les étudiants de droit et d'AES avaient trois UE à valider à la fin du premier semestre alors que les étudiants inscrits en psychologie en avaient quatre. Dans chacune des filières, les corrélations entre les notes des UE sont élevées (le plus souvent supérieure à $+0,70$ ) et permettent de considérer la note moyenne 
Tableau 5. Modèles généraux analysant la variance des notes au premier semestre $(\mathbf{N}=612)$

\begin{tabular}{|c|c|c|c|c|c|c|c|}
\hline \multirow[b]{3}{*}{ Modalité de référence } & \multirow[b]{3}{*}{ Modalité active } & \multirow{2}{*}{\multicolumn{2}{|c|}{ Modèle 4}} & & & & \\
\hline & & & & \multicolumn{2}{|c|}{ Modèle 5} & \multicolumn{2}{|c|}{ Modèle 6} \\
\hline & & Coef. & Prob. & Coef. & Prob. & Coef. & Prob. \\
\hline \multicolumn{2}{|l|}{ Constante } & $+3,8$ & 0,00 & $+2,1$ & 0,00 & $+2,2$ & 0,00 \\
\hline \multicolumn{8}{|l|}{ Filière fréquentée } \\
\hline \multirow{2}{*}{ Droit } & AES & $+2,4$ & 0,00 & $+2,4$ & 0,00 & $+2,4$ & 0,00 \\
\hline & Psychologie & $+2,5$ & 0,00 & $+2,5$ & 0,00 & $+2,5$ & 0,00 \\
\hline Garçon & Fille & $+0,2$ & 0,33 & $+0,2$ & 0,39 & $+0,2$ & 0,41 \\
\hline Retard scolaire & Âge normal & $+0,6$ & 0,00 & $+0,7$ & 0,00 & $+0,7$ & 0,00 \\
\hline Non boursier & Boursier & $-0,4$ & 0,02 & $-0,4$ & 0,02 & $-0,4$ & 0,03 \\
\hline \multicolumn{8}{|l|}{ Série du bac } \\
\hline \multirow{4}{*}{ Bac professionnel } & Bac technologique & $+0,9$ & 0,03 & $+0,7$ & 0,09 & $+0,7$ & 0,09 \\
\hline & Bac L & $+2,7$ & 0,00 & $+2,3$ & 0,00 & $+2,3$ & 0,00 \\
\hline & Bac ES & $+3,2$ & 0,00 & $+2,9$ & 0,00 & $+2,9$ & 0,00 \\
\hline & Bac S & $+4,4$ & 0,00 & $+3,9$ & 0,09 & $+4,0$ & 0,00 \\
\hline Pas de mention & Mention AB, B ou TB & $+2,3$ & 0,00 & $+2,1$ & 0,00 & $+2,1$ & 0,00 \\
\hline \multicolumn{2}{|c|}{ Score en compréhension de l'écrit (DALF) } & & & $+0,15$ & 0,00 & $+0,15$ & 0,00 \\
\hline \multicolumn{2}{|c|}{ Score au Raven (\% de réussite) } & & & & & $-0,00$ & 0,68 \\
\hline \multicolumn{2}{|l|}{$\mathrm{R}^{2}$} & \multicolumn{2}{|c|}{0,40} & \multicolumn{2}{|c|}{0,43} & \multicolumn{2}{|c|}{0,43} \\
\hline
\end{tabular}

Figure 2. Effets nets de la série du bac sur la note moyenne du premier semestre

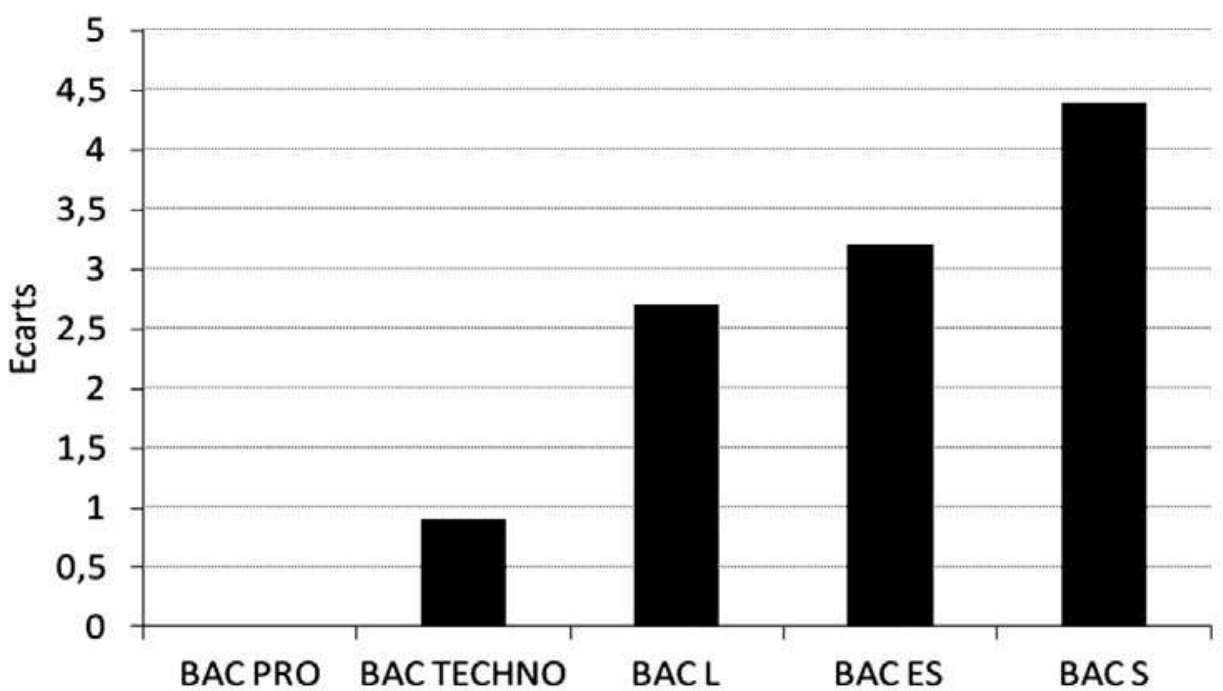


du premier semestre comme un indicateur global des performances des étudiants. Les moyennes des notes globales du semestre 1 sont proches de 9 sur 20 dans les trois filières. Ces notes des UE des trois filières ne sont pas comparables dans la mesure où les enseignements qui les composent ne sont pas les mêmes, on ne peut donc pas comparer les degrés de réussite des étudiants selon la filière. Cela a pour conséquence que l'utilisation de cette note moyenne sur l'ensemble de l'échantillon devra tenir compte de ce biais ; c'est pourquoi nous introduirons systématiquement la filière fréquentée comme variable de contrôle dans les modèles de régression. Ceci permettra de mesurer l'influence des caractéristiques des étudiants en raisonnant à « filière fréquentée identique ».

Les premiers facteurs examinés sont les capacités cognitives, plusieurs modèles successifs ont été estimés en faisant intervenir les différentes mesures de cette dimension (modèles non présentés). Les performances en mémoire de travail et en vitesse de traitement n'exercent aucun impact sur les résultats universitaires ; en revanche, on relève un effet positif du score obtenu au Raven (le coefficient est positif et significatif). À titre d'illustration, une augmentation de $25 \%$ du taux de réussite au Raven entraîne un gain de 0,5 point sur la note moyenne, ce qui n'est pas négligeable. Des modèles globaux (tableau 5) ont ensuite été estimés en intégrant l'ensemble des caractéristiques des étudiants, celles-ci expliquent $40 \%$ de la variance des notes de fin de premier semestre (modèle 4). Si aucune différence n'est relevée entre garçons et filles, l'âge exerce un impact significatif (réussite moindre pour les étudiants ayant accumulé du retard). De même, le fait d'être boursier, qui d'une certaine manière rend compte d'une caractéristique sociale de l'étudiant, est associé à une moindre réussite (près d'un demi-point de moins, en moyenne).

La série du bac est le facteur de loin le plus discriminant. Cette variable explique à elle seule $24 \%$ de la variance des notes. La lecture des modèles montre une nette hiérarchie des séries avec les performances les plus faibles relevées pour les bacs professionnels. La série $S$ est associée aux résultats les meilleurs, suivie de la série ES, puis de la série L. Les écarts sont très élevés puisque 4 points séparent les bacs professionnels des bacs scientifiques (figure 2). À série du bac équivalente, la possession d'une mention, quelle qu'elle soit, crée aussi des différences : les étudiants qui en sont titulaires obtiennent 2 points de plus que les autres. Au total, le passé scolaire reste un élément déterminant pour le début du parcours universitaire. Ainsi, entre un étudiant venant d'un bac professionnel avec du retard scolaire et un étudiant possédant un bac scientifique avec mention, on relève un écart de plus de 6,5 points sur la moyenne de fin de premier semestre. On met à nouveau en évidence à travers ces résultats l'importance du parcours scolaire antérieur pour la réussite à l'université (Prouteau, 2009).

Le modèle 5 apporte une information importante, puisque, à la marge de l'influence de ces facteurs classiques, le niveau académique exerce un effet autonome sur les résultats des étudiants. La valeur du coefficient $(+0,15)$ indique qu'entre deux étudiants ayant un écart-type de différence au score du DALF (l'écart-type du score au DALF vaut 3,6 points) la différence à la moyenne du premier semestre s'évalue à plus d'un demi-point $\left(3,6^{\star} 0,15\right)$. Les performances en raisonnement logique n'ont pas d'effet spécifique sur les résultats scolaires. Le coefficient associé à la variable "Score au Raven " a une valeur nulle (modèle 6). Les capacités cognitives des étudiants, telles qu'elles ont été mesurées dans la présente étude ${ }^{5}$, n'exercent donc pas d'effet autonome sur les performances universitaires ; en référence aux analyses exposées précédemment, on en déduit que ces capacités se sont exprimées essentiellement au cours de la scolarité passée.

Une manière complémentaire de mesurer la réussite en fin de premier semestre est de raisonner en termes de probabilité de réussite ou d'échec. Sur l'échantillon, la proportion d'étudiants ayant validé le semestre (ceux qui obtiennent au moins 10 sur 20) est de $32 \%$. Un modèle de régression logistique a été estimé (tableau 6) qui intègre les mêmes facteurs explicatifs ; ce modèle présente des résultats proches des précédents.

Les chiffres mentionnés dans la colonne «Exp. » du modèle 7 expriment les rapports de cote (odds ratio) associés aux coefficients, ils indiquent qu'un étudiant titulaire d'un bac $S$ a 10,5 fois plus de chance d'être admis au semestre 1 qu'un étudiant titulaire d'un bac professionnel ; de même, un étudiant ayant obtenu une mention au bac a 5,1 fois plus de chances d'être admis qu'un non titulaire d'une mention. Le score au DALF exerce son influence puisque les étudiants ayant des performances élevées dans ce domaine ont plus de chances de réussir que les autres. En revanche, les capacités cognitives mesurées par le Raven n'apparaissent pas être un facteur autonome discriminant.

Au-delà des variables mobilisées jusqu'à présent, d'autres informations collectées à partir d'un questionnaire ont permis d'intégrer aux analyses des dimensions plus subjectives dans les conditions d'accès à 
Tableau 6. Modèle logistique de réussite au premier semestre $(\mathrm{N}=612)$

\begin{tabular}{|c|c|c|c|c|}
\hline & & & & \\
\hline & & \multicolumn{3}{|c|}{ Modèle 7} \\
\hline Modalité de référence & Modalité active & Coef. & Prob. & Exp. \\
\hline \multicolumn{2}{|l|}{ Constante } & $-5,2$ & 0,00 & 4,8 \\
\hline \multicolumn{5}{|l|}{ Filière fréquentée } \\
\hline \multirow{2}{*}{ Droit } & AES & $+2,0$ & 0,00 & 7,2 \\
\hline & Psychologie & $+1,3$ & 0,00 & 3,8 \\
\hline Garçon & Fille & $+0,0$ & 0,81 & 1,1 \\
\hline Retard scolaire & Âge normal & $+0,5$ & 0,06 & 1,6 \\
\hline Non boursier & Boursier & $-0,2$ & 0,34 & 0,8 \\
\hline \multicolumn{5}{|l|}{ Série du bac } \\
\hline \multirow{4}{*}{ Bac professionnel } & Bac technologique & $-0,4$ & 0,44 & 0,6 \\
\hline & Bac L & $+1,0$ & 0,05 & 2,8 \\
\hline & Bac ES & $+1,4$ & 0,00 & 4,3 \\
\hline & Bac S & $+2,6$ & 0,00 & 10,5 \\
\hline Pas de mention & Mention AB, B ou TB & $+1,6$ & 0,00 & 5,1 \\
\hline \multicolumn{2}{|c|}{ Score en compréhension de l'écrit (DALF) } & $+0,10$ & 0,00 & 1,1 \\
\hline \multicolumn{2}{|c|}{ Score au Raven (\% de réussite) } & $+0,00$ & 0,28 & 1,0 \\
\hline \multicolumn{2}{|l|}{$\mathrm{R}^{2}$ de Nagelkerke } & \multicolumn{3}{|c|}{0,37} \\
\hline
\end{tabular}

Lecture : le modèle de régression logistique a la même finalité qu'un modèle de régression linéaire mais il s'utilise quand la variable dépendante est dichotomique, soit dans notre exemple « réussite » ou "échec " au premier semestre. À chaque modalité des variables indépendantes est associé un coefficient dont l'interprétation est basée sur les rapports de chance et non sur sa valeur directe.

l'université. C'est notamment le cas des choix d'orientation après le bac. Sur l'échantillon, $58 \%$ déclarent avoir choisi leur filière en fonction d'un projet professionnel, 48 \% par intérêt ou par goût, $13 \%$ par curiosité, $5 \%$ par défaut et seulement $3 \%$ sur la base de la réussite probable dans les disciplines choisies. On notera que les choix multiples sont relativement limités puisque $22 \%$ des étudiants mentionnent deux choix, $2 \%$ trois choix et seulement $0,5 \%$ quatre choix. Parmi les différentes modalités, une seule exerce une influence significative sur la moyenne du premier semestre, il s'agit de l'orientation décidée en fonction d'un choix professionnel. Quand on intègre cette variable au modèle 6 précédent, on relève un effet positif et significatif (au seuil de $1 \%$ ) de cette modalité de choix d'orientation. Les étudiants qui déclarent avoir décidé de leur avenir scolaire sur la base d'un projet professionnel ont, toutes choses égales par ailleurs, une note moyenne supérieure de près d'un demi-point aux autres (coefficient de $+0,49$ ). L'adéquation de l'orientation à un projet professionnel contribue donc pleinement à la réussite même si les filières concernées dans notre étude ne sont sans doute pas celles pour lesquelles ce lien est susceptible d'être le plus fort, notamment en référence aux filières "appliquées".

\section{L'ANALYSE DE LA RÉUSSITE EN FIN D'ANNÉE UNIVERSITAIRE}

Ces estimations porteront sur des effectifs plus réduits (69 étudiants ont abandonné après les partiels de janvier), les faibles résultats aux examens de 


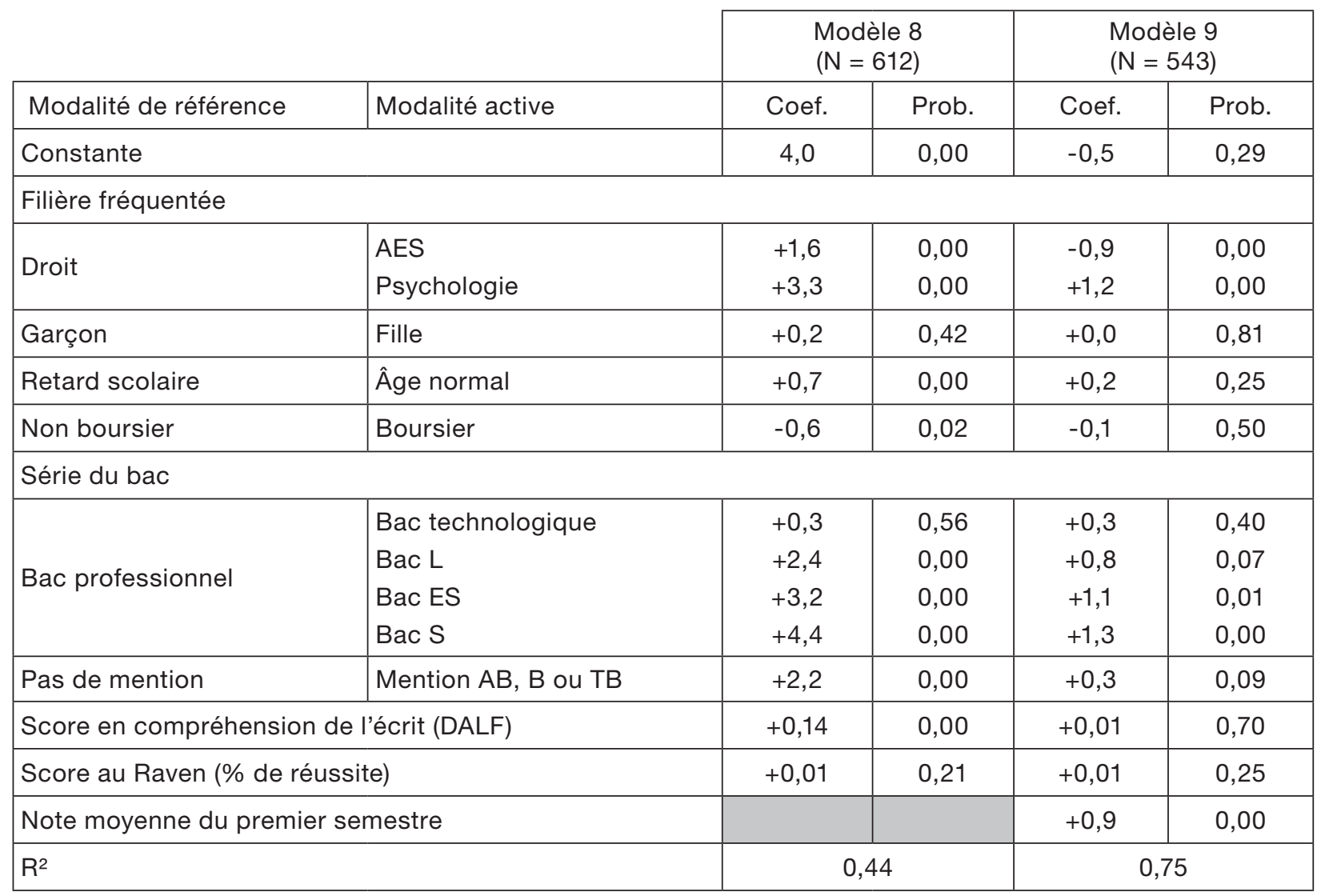

janvier semblent être le facteur le plus déterminant de l'abandon puisque les étudiants concernés avaient une moyenne de 5,4 sur 20 à l'issue du premier semestre. Un premier constat est la très forte corrélation existant entre les deux prises d'informations (premier et second semestre), bien que les disciplines évaluées ne soient pas les mêmes (les UE des deux semestres sont différentes), le classement des étudiants n'évolue guère puisque la corrélation relevée sur l'ensemble de l'échantillon est de $+0,83$ et ne varie guère dans les trois filières : $+0,85$ en droit, $+0,85$ en psycho et $+0,84$ en AES. C'est donc près de $70 \%$ de la variance des notes du second semestre qui est expliquée par les performances du premier semestre. Le modèle 9 (tableau 7), qui analyse les déterminants de la réussite au second semestre, produit des estimations comparables à celles relatives au semestre précédent, la série du bac, la mention, l'âge apparaissent toujours comme des facteurs déterminants de la réussite. On relèvera également l'influence positive des performances en compréhension de l'écrit et l'absence d'effet du score en raisonnement logique. Par ailleurs, le choix d'orientation n'exerce pas non plus d'effet sur les résultats au second semestre (modèle non présenté).

Bien que les échelles de mesure soient différentes, nous avons estimé un modèle de progression (modèle 9) entre les résultats des deux semestres. En effet, ce modèle fournit une indication sur les progrès relatifs des étudiants en cours d'année en termes de position au sein de l'échantillon. Le modèle explique les trois quarts de la variance des notes du second semestre. Certaines variables affichent des coefficients significatifs : mention et série du bac. II reste à présenter les résultats de l'ensemble de l'année universitaire : notes moyennes de l'année (modèle 11) et décision de fin d'année (modèle 12, non présenté). La moyenne générale sur l'année est de 9,4 avec un écart-type de 3 . Les estimations du modèle 11 confirment les analyses précédentes, on relèvera cependant 
Tableau 8. Modèle analysant la variance des notes de l'année ( $N=543)$

\begin{tabular}{|c|c|c|c|}
\hline \multirow[b]{2}{*}{ Modalité de référence } & \multirow[b]{2}{*}{ Modalité active } & \multicolumn{2}{|c|}{ Modèle 11} \\
\hline & & Coef. & Prob. \\
\hline \multicolumn{2}{|l|}{ Constante } & 2,8 & 0,00 \\
\hline \multicolumn{4}{|l|}{ Filière fréquentée } \\
\hline \multirow{2}{*}{ Droit } & AES & $+2,2$ & 0,00 \\
\hline & Psychologie & $+2,9$ & 0,00 \\
\hline Garçon & Fille & $+0,2$ & 0,38 \\
\hline Retard scolaire & Âge normal & $+0,7$ & 0,00 \\
\hline Non boursier & Boursier & $-0,5$ & 0,02 \\
\hline \multicolumn{4}{|l|}{ Série du bac } \\
\hline \multirow{3}{*}{$\begin{array}{l}\text { Bac professionnel } \\
\text { Bac technologique }\end{array}$} & Bac L & $+1,9$ & 0,00 \\
\hline & Bac ES & $+2,7$ & 0,00 \\
\hline & Bac $S$ & $+3,7$ & 0,00 \\
\hline Pas de mention & Mention AB, B ou TB & $+2,1$ & 0,00 \\
\hline \multicolumn{2}{|c|}{ Score en compréhension de l'écrit (DALF) } & $+0,14$ & 0,00 \\
\hline \multicolumn{2}{|c|}{ Score au Raven (\% de réussite) } & $+0,00$ & 0,47 \\
\hline \multicolumn{2}{|l|}{$\mathrm{R}^{2}$} & \multicolumn{2}{|c|}{0,47} \\
\hline
\end{tabular}

Figure 3. Analyse en pistes causales mettant en relation le passé scolaire, les performances académiques, les capacités cognitives et les résultats en fin de première année universitaire $(\mathrm{N}=543)$



Lecture : les modèles d'analyse en pistes causales ont l'intérêt de mesurer des effets directs et indirects des variables dans un même modèle. Les effets directs correspondent à la valeur du coefficient de régression de la variable indépendante (VI) sur la variable dépendante (VD). On calcule les effets indirects en faisant le produit des coefficients des pistes que l'on doit parcourir pour faire le chemin qui part de la VI et va jusqu'à la VD en passant par des variables intermédiaires, d'où l'appellation de «pistes causales ». Les corrélations sont significatives au seuil de $1 \%$.

que les performances des étudiants des bacs technologiques et professionnels ne se distinguent plus (tableau 8).

De façon à mieux comprendre les relations statistiques existant entre les différentes familles de variables qui nous intéressent dans cette recherche, un modèle en path analysis (modèle de cheminement) a été élaboré. Il permet de mettre à jour les effets directs ou indirects du passé scolaire et des capacités cognitives sur les résultats obtenus en fin d'année.

À la lumière de la figure 3 , le premier constat porte sur l'effet du passé scolaire sur les résultats en fin d'année, cet effet s'exerçant de façon directe (coefficient très significatif de 0,28 ), mais aussi de façon 


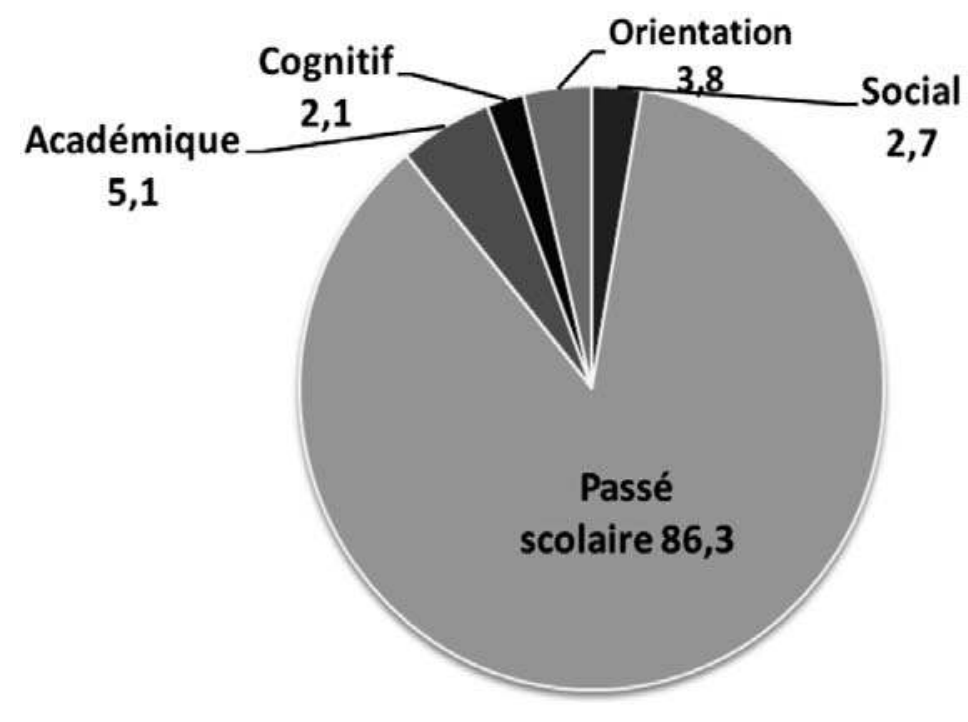

indirecte en transitant par les capacités cognitives $\left(0,49^{\star} 0,03\right)$. Ainsi, le passé scolaire (mention et type de bac) exerce un fort impact sur les résultats obtenus au Raven qui eux-mêmes influent par la suite, mais de façon très modérée $(0,03)$, sur la réussite en fin d'année. De façon similaire, le passé scolaire de l'étudiant influence fortement les résultats au test du DALF, les compétences cognitives exercent quant à elles un effet quatre fois moins important sur les résultats obtenus au DALF. Ces performances académiques auront elles-mêmes un effet direct mais très faible sur les résultats obtenus en fin d'année. Nous nous sommes enfin intéressés conjointement à l'ensemble des déterminants de la réussite en fin de première année avec une régression logistique et un phénomène intéressant apparaît. L'effet du niveau académique (score en compréhension de l'écrit) s'atténue puisque le coefficient attaché à cette variable n'est significatif qu'au seuil de $8 \%$. En revanche, les performances au Raven ont tendance à exercer une influence positive sur les résultats, la probabilité d'erreur associée au coefficient étant de $10 \%$. On ne peut bien sûr ici parler que de tendance puisque le seuil des $5 \%$ d'erreur n'est pas atteint. Une autre information est fournie dans ce modèle ; les modalités de choix d'orientation universitaire jouent un rôle sur la réussite (ce qui n'était pas le cas dans les estimations précédentes relatives aux notes de fin d'année) : les étudiants qui ont effectué leur choix en fonction d'un projet professionnel ont une probabilité de réussite supérieure de 1,7 fois à celle des autres étudiants. Sur la base des analyses réalisées, on peut tenter de rendre compte globalement du poids de chaque groupe de facteurs sur la réussite, on obtient alors la situation visualisée par la figure 4 dont les données découlent du modèle logistique présenté auparavant (modèle 12) et d'autres modèles intermédiaires qui intègrent successivement les groupes de variables explicatives. II est alors possible de chiffrer la contribution propre de chacun de ces groupes à l'explication des écarts de réussite entre étudiants. L'ensemble des variables explique environ $30 \%$ de la variance des résultats (en déduisant la contribution de l'appartenance à une filière) ; la figure 4 décompose cette variance en termes de pourcentages selon les différents groupes de facteurs.

On soulignera encore une fois le poids très fort du passé scolaire ( $86,3 \%$ de la variance expliquée), le niveau académique (DALF) représentant $5 \%$ de cette même variance, les modalités de choix d'orientation $3,8 \%$, les caractéristiques sociales (le fait d'être boursier ou non) moins de $3 \%$ et les performances cognitives (Raven) seulement un peu plus de $2 \%$.

\section{CONCLUSION}

Au terme de cet article, les résultats exposés demandent à être davantage commentés au risque 
de laisser la place à des possibilités d'interprétations confuses, voire erronées. Un premier point est de rappeler que cette étude novatrice dans le contexte français présente certaines limites. La principale concerne l'échantillon sur lequel ont porté les analyses statistiques. Celui-ci ne représente qu'une partie de la population des jeunes s'orientant vers des études post-bac et il est possible que certains effets ou relations détectés dans cette recherche ne soient pas totalement généralisables, notamment à la population d'étudiants inscrits dans les filières scientifiques ou plus "prestigieuses » (comme les grandes écoles) mais aussi aux bacheliers s'orientant vers un BTS ou un DUT. On peut alors supposer que la structure des relations entre facteurs sociaux et cognitifs diffère selon le public visé. À ce titre, la différence entre filles et garçons observée dans les performances en mémoire de travail peut être une illustration de cette spécificité de l'échantillon et des biais de sélection qui peuvent y être associés. Une autre limite a trait aux outils utilisés pour la mesure des compétences et capacités des étudiants. Si les tests cognitifs de raisonnement logique, de vitesse de traitement de l'information et de mémoire de travail présentent une forte validité et sont basés sur des fondements théoriques solides, on doit reconnaître que la mesure des compétences académiques est partielle et limitée, faute de disposer d'autres outils davantage adaptés à la population concernée.

Un second point a trait à l'interprétation même des résultats exposés. Nous avons insisté sur l'importance des facteurs liés au parcours scolaire antérieur pour expliquer les différences de réussite des étudiants. En effet, le retard scolaire, la série et la mention au bac sont des éléments qui hiérarchisent nettement les performances à l'issue de la première année passée à l'université et cette constatation, non originale, est partagée par d'autres travaux sur l'enseignement supérieur. Mais le point qui fait débat, à la base de nos questions de recherche, est l'influence des capacités cognitives et des compétences académiques sur les notes obtenues à l'université. Les résultats montrent clairement le rôle limité de ces deux dimensions dans l'explication des écarts de réussite entre les étudiants quand on raisonne à parcours scolaire et à caractéristiques socio-démographiques donnés. Or, nous avons aussi mis en évidence lors des premières analyses présentées dans ce texte l'influence des caractéristiques du passé scolaire sur les performances académiques et cognitives ; sans que nous puissions le vérifier sur ces données, nous pouvons néanmoins supposer sans risque, en accord avec les nombreuses recherches sociologiques sur les déterminants de la réussite, que les facteurs socio-économiques et culturels (milieu familial) ont été des déterminants importants de la qualité de ce passé scolaire en primaire, au collège et au lycée. Le fait de ne pas trouver d'impact massif et autonome des compétences académiques et des capacités cognitives en fin de première année universitaire montre bien que l'essentiel de l'influence de ces facteurs s'est exprimé auparavant tout au long de la scolarité. On peut aussi supposer que les examens des différentes disciplines évaluées dans les trois filières considérées mobilisent davantage la restitution de connaissances plutôt que la mise en œuvre de processus cognitifs de haut niveau sur le plan de la taxonomie des objectifs. Enfin, un certain nombre de facteurs propres aux étudiants, qui feront l'objet d'un second volet de la recherche, n'ont pas été pris en compte. Ainsi, l'investissement personnel en temps de travail et la motivation devraient sans doute contribuer à expliquer une part des différences individuelles de parcours.

En conclusion, il apparaît que la réussite des étudiants est très dépendante de la nature de la scolarité passée et ces résultats interrogent plus largement sur les procédures d'orientation et les choix d'études à l'issue de l'enseignement secondaire. II est important de relever que les étudiants qui ont choisi leur filière sur la base d'un projet professionnel réussissent mieux que les autres. Au-delà des forts effets liés à la série du baccalauréat, l'influence autonome du niveau de compétence en compréhension de l'écrit montre que les compétences acquises, quelle que soit la filière fréquentée dans l'enseignement secondaire, contribuent à la réussite à l'université. Enfin, si les capacités cognitives ne semblent pas, à l'issue de cette recherche, exercer un effet autonome sur les résultats des étudiants, il reste à démontrer que cela reste vrai dans la suite du parcours universitaire au niveau de la licence ou du master.

Sophie Morlaix sophie.morlaix@u-bourgogne.fr Université de Bourgogne, IREDU-CNRS

Bruno Suchaut bruno.suchaut@vd.ch Unité de recherche pour le pilotage des systèmes pédagogiques (URSP) 
1 Ce travail s'inscrit dans le cadre d'une recherche financée par l'Agence nationale de la recherche (ANR) et conduite en collaboration avec le LEAD-CNRS. Les auteurs tiennent à remercier Valérie Camos, Prune Lagner et Marielle Lambert pour leur investissement, à différents niveaux, dans cette recherche.

2 Des informations sur les caractéristiques socio-démographiques des étudiants et sur leur passé scolaire ont été extraites des bases APOGEE de l'université de Bourgogne. Certaines autres informations ont été collectées sur la base d'un questionnaire spécifique, c'est notamment le cas pour les choix d'orientation réalisés après le baccalauréat.
3 Chaque score est la moyenne centrée réduite des scores centrés réduits obtenus à chaque épreuve.

4 Les corrélations négatives viennent du fait que l'échelle des scores de vitesse de traitement est différente des deux autres échelles (Raven et mémoire de travail) puisque, pour cette dimension, les scores les plus élevés correspondent aux performances les plus faibles.

5 Dans un contexte académique et avec d'autres outils, ce lien a pu être établi (Wathelet, Vieillevoye \& Romainville, 2012).

\section{BIBLIOGRAPHIE}

BEAUPÈRE N. \& BOUDESSEUL G. (2009). Sortir sans diplôme de l'Université. Comprendre les parcours d'étudiants "décrocheurs ». Paris: La Documentation française, coll. "Études \& recherche".

BÉDUWÉ C. \& GIRET J.-F. (2004). " Le travail en cours d'études a-t-il une valeur professionnelle? ». Économie et statistique, no 378-379, p. 55-83.

BARROUILLET P., BERNARDIN S., PORTRAT S., VERGAUWE E. \& CAMOS V. (2007). "Time and cognitive load in working memory ". Journal of Experimental Psychology: Learning, Memory and Cognition, n० 33-3, p. 570-585.

BARROUILLET P., CAMOS V., MORLAIX S. \& SUCHAUT B. (2008). "Progressions scolaires, mémoire de travail et origine sociale: quels liens à l'école élémentaire? ". Revue française de pédagogie, no 162, p. 5-14.

BARROUILLET P., PORTRAT S., VERGAUWE E., DIEPENDAELE K. \& CAMOS V. (2011). "Further evidence for temporal decay in working memory ". Journal of Experimental Psychology : Learning, Memory and Cognition, no 37, p. 1302-1317.

BORRAS I. (2011). " Le tutorat à I'université : peut-on forcer les étudiants à la réussite ? ". Bref du CEREQ, n०290.

COULON A. (1997). Le métier d'étudiant: l'entrée dans la vie universitaire. Paris : PUF.

DANNER M. (2000). "À qui profite le tutorat mis en place dans le premier cycle universitaire? ". Les Sciences de l'éducation pour l'ère nouvelle, no 1, p. 25-41.

DANEMAN M. \& CARPENTER P.A. (1980). «Individual differences in working memory and reading ". Journal of Verbal Learning and Verbal Behavior, no 19, p. 450466.

DURU-BELLAT M. (1995). « Des tentatives de prédiction aux écueils de la prévention en matière d'échec scolaire en première année d'université ". Savoir éducation formation, n० 3, p. 399-416.

FRICKEY A. \& PRIMON J.-L. (2002). « Jeunes issus de l'immigration : les diplômes de l'enseignement supé- rieur ne garantissent pas un égal accès au marché du travail ». Formation emploi, no 79, p. 19-107

HUTEAU M. \& LAUTREY J. (1999). Évaluer l'intelligence. Psychométrie cognitive. Paris: PUF.

JENSEN A.R. (1987). "Individual differences in the Hick paradigm ». In P.A. Vernon (dir.), Speed of information processing and intelligence. Norwood, NJ : Ablex, p. 101-175.

LÉPINE R., BARROUILLET P. \& CAMOS V. (2005). « What makes working memory spans so predictive of high level cognition? ». Psychonomic Bulletin and Review, no 12-1, p. 165-170.

MINISTÈRE DE L'ÉDUCATION NATIONALE (2010). Repères et références statistiques sur les enseignements, la formation et la recherche. Vanves : Ministère de l'Éducation nationale.

MICHAUT C. (2003). "L'efficacité des dispositifs d'aide aux étudiants dans les universités ". Recherche et formation, no 43, p. 101-113.

PROUTEAU D. (2009). "Parcours et réussite en licence des inscrits en L1 en 2004 ». Note d'information de la $D P D, \mathrm{n} \circ 23$, p. 1-6.

ROMAINVILLE M. (2000). L'échec dans l'université de masse. Paris : L'Harmattan.

SHANKLAND R. (2009). Pédagogies nouvelles et compétences psychosociales. Paris : L'Harmattan.

SHULRUF B., HATTIE J. \& TUMEN S. (2008). « The predictability of enrolment and first $\mathbb{S}$ Studies in Higher Education, no 33-6, p. 685-698.

TURNER M.L. \& ENGLE R.W. (1989). «Is working memory capacity task dependent? ». Journal of Memory and Language, no 28, p. 127-154.

WATHELET V., VIEILLEVOYE S. \& ROMAINVILLE $M$. (2012). "Maîtrise des prérequis et réussite à l'université ". In M. Romainville \& C. Michaut, Réussite, échec et abandon dans l'enseignement supérieur. Bruxelles: De Boeck, coll. «Perspectives en éducation \& formation », p. 221-250. 
ANNEXE. COMPARAISON DE LA POPULATION D'ÉTUDIANTS DES TROIS FILIÈRES (AES, DROIT ET PSYCHOLOGIE) PAR RAPPORT À L’ENSEMBLE DES ÉTUDIANTS INSCRITS EN PREMIÈRE ANNÉE À L'UNIVERSITÉ DE BOURGOGNE (EN POURCENTAGE)

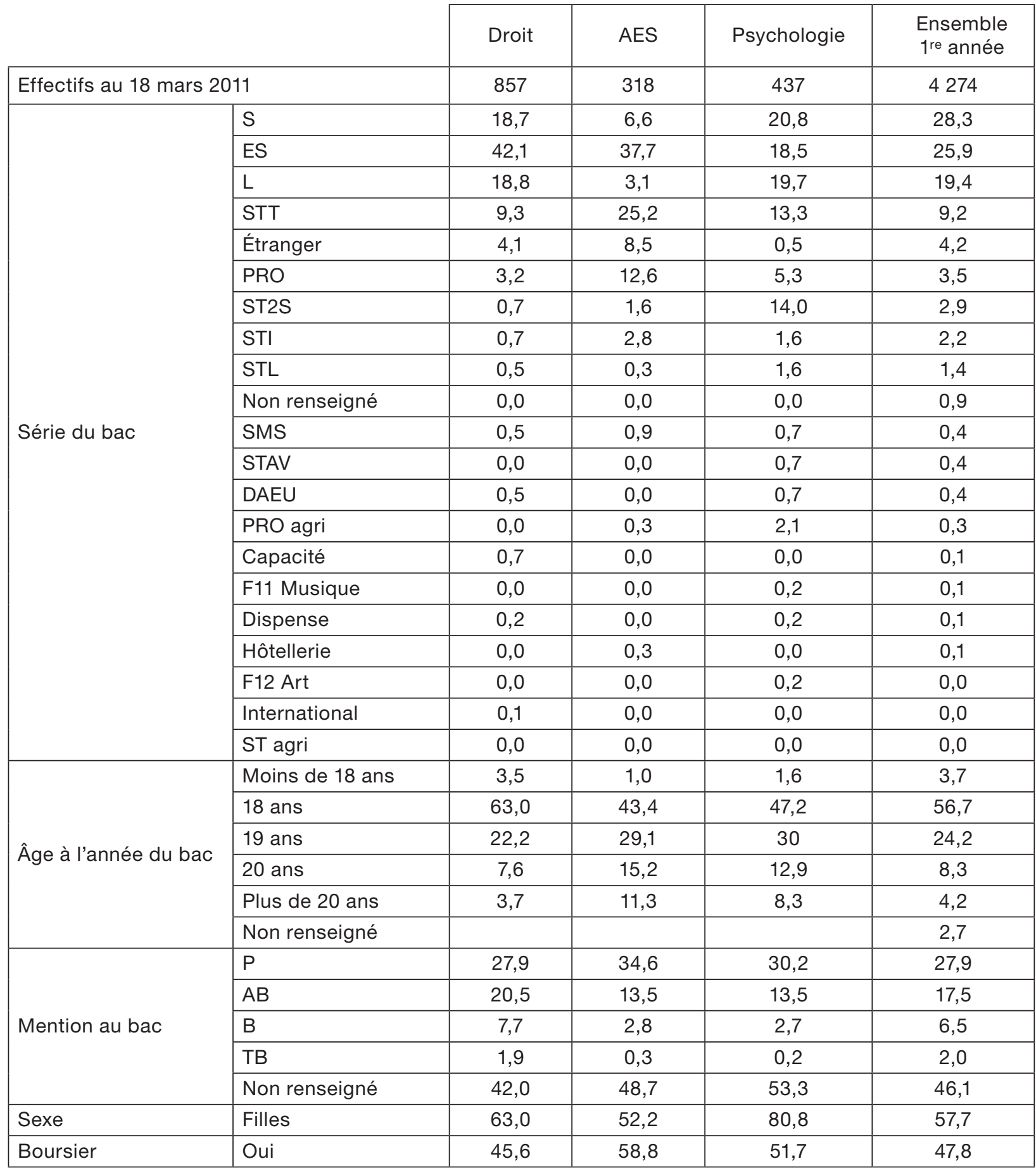




\begin{tabular}{|l|l|c|c|c|c|}
\hline & $\begin{array}{l}\text { Agriculteurs } \\
\text { exploitants }\end{array}$ & 3,5 & 1,6 & 3,0 & 3,2 \\
\cline { 2 - 5 } & $\begin{array}{l}\text { Artisans, } \\
\text { commerçants, } \\
\text { chefs d'entreprise }\end{array}$ & 9,2 & 8,2 & 8,5 & 8,3 \\
\cline { 2 - 6 } & $\begin{array}{l}\text { Cadres } \\
\text { et professions } \\
\text { intellectuelles }\end{array}$ & 26,1 & 13,5 & 16,2 & 21,8 \\
\cline { 2 - 6 } & $\begin{array}{l}\text { Professions } \\
\text { Profession } \\
\text { et catégorie } \\
\text { socioprofessionnelle }\end{array}$ & 14,4 & 9,1 & 17,2 & 15,0 \\
\cline { 2 - 6 } & Employés & 14,4 & 18,2 & 15,1 & 14,5 \\
\cline { 2 - 6 } & Ouvriers & 16,9 & 24,8 & 22,0 & 19,1 \\
\cline { 2 - 6 } & Retraités & 6,9 & 10,7 & 6,4 & 3,1 \\
\cline { 2 - 6 } & $\begin{array}{l}\text { Sans activités } \\
\text { professionnelles }\end{array}$ & 4,4 & 5,7 & 3,2 & 7,4 \\
\cline { 2 - 6 } & Non renseigné & 4,2 & 8,2 & 8,5 & 3,6 \\
\hline
\end{tabular}

